\title{
An X-ray Absorption Spectroscopy study of the interaction of different quartz surfaces with $\mathrm{Fe}$ (II) and $\mathrm{Fe}$ (III) species
}

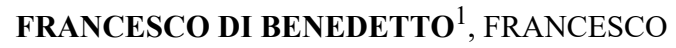 \\ D'ACAPITO $^{2}$, GIORDANO MONTEGROSSI ${ }^{2}$ AND \\ MASSIMO INNOCENTI ${ }^{3}$
}

${ }^{1}$ University of Ferrara

${ }^{2} \mathrm{CNR}$

${ }^{3}$ University of Florence

Presenting Author: francesco.dibenedetto@unife.it

The specific reactivity of silica surfaces towards Fe species in solution is the object of a large interest aimed at unravelling, at the molecular scale, the processes under which silica can initiate and propagate oxidative stress to the lung tissues. In particular, the Fe species, their interchange and their stability are involved in the Fenton cycle, a chemical catalytic process able to increase the number of Reactive Oxygen Species (ROS) in a given environment [1]. The present study is aimed at assessing if different surfaces of $\alpha$-quartz crystals, chosen as the most relevant silica polymorph in terms of health hazard, can variably interact with $\mathrm{Fe}(\mathrm{II})$ and $\mathrm{Fe}(\mathrm{III})$ solutions under laboratory conditions.

Three different natural single crystal of $\alpha$-quartz, having centimetric size, where cut and polished along three main directions: (101), (100), (001). These cleaned surfaces were let interact with standard solutions of $\mathrm{Fe}(\mathrm{II})$ and $\mathrm{Fe}(\mathrm{III})$, then dried and analysed through different techniques: Atomic Force Microscopy, to investigate the evolution of the surface roughness, and X-ray Absorption Spectroscopy (performed in the Grazing Incident configuration), to assess the Fe speciation at the crystal surface. AFM characterizations were carried out before and after the chemical treatment. The results were then compared with the results of the thermochemical modelling of the system, carried out by the use of Pourbaix prevailing charts calculated using the PHREEQC software.

The preliminary results point to a behaviour comparatively homogeneous of the different investigated surfaces with respect to the formation of Fe-oxy(hydro)xides at the interface. Fe-O-Si configurations were not detected by ReflEXAFS, and the final products seems, in some way, driven mainly by thermodynamical constraints of the solution, at least concerning the prevailing species. Conversely, a modest but not negligible effect of the different leaching treatment seem able to gain differential development of a long range ordered structure.

- $\quad$ Pavan et al. (2020) PNAS, 117(45), 27836-27846 